ORIGINAL ARTICLE

\title{
A population based study of herpes simplex virus 2 seroprevalence in rural Costa Rica
}

\author{
A C Rodríguez, P E Castle, J S Smith, C Bratti, A Hildesheim, M Schiffman, R Viscidi, R D Burk, \\ $\mathrm{R} L$ Ashley, X Castellsagué, R Herrero
}

Sex Transm Infect 2003;79:460-465

See end of article for authors' affiliations

.....................

Correspondence to:

Dr Ana Cecilia Rodríguez, Proyecto Epidemiológico Guancaste, Apdo 1811250, Escazú, Costa Rica; project@racsa.co.cr

Accepted for publication 9 May 2003
Objectives: To determine seroprevalence and determinants of herpes simplex virus 2 (HSV-2) seropositivity, in a random sample of a population based cohort of 10049 women of Guanacaste, Costa Rica, using a highly sensitive and specific serological assay.

Methods: Seroprevalence was determined by a type specific HSV-2 ELISA assay in an age stratified random sample of 1100 women. Univariate and multivariate logistic regression was used to calculate odds ratios and $95 \%$ confidence intervals for risk factors of seropositivity.

Results: Overall age adjusted HSV-2 seroprevalence was $38.5 \%(95 \% \mathrm{Cl}, 37.5$ to 39.5$)$, and it was strongly associated with increasing age $\left(\mathrm{p}_{\text {Trend }}<0.0001\right)$, both among monogamous women and women with multiple sexual partners. A greater number of lifetime sexual partners increased the risk of seropositivity, with a $28.2 \%(95 \% \mathrm{Cl}, 24.4$ to 32.2$)$ seroprevalence among monogamous women and $75 \%$ (95\% Cl, 65.6 to 83.0 ) seroprevalence for those with four or more partners $(\mathrm{OR}=7.695 \% \mathrm{Cl}, 4.7$ to 12.4 $\left.\mathrm{P}_{\text {Trend }}<0.0001\right)$. Barrier contraceptive use was negatively associated with HSV-2 seropositivity (OR 0.54 , $95 \% \mathrm{Cl}, 0.31$ to 0.94$)$. Women with antibodies against HPV 16, 18, or 31 were 1.6 times more likely to be HSV-2 seropositive (OR 1.6, 95\% Cl, 1.2 to 2.1 ).

Conclusions: HSV-2 infection is highly endemic in Guanacaste, even among lifetime monogamous women, suggesting a role of male behaviour in the transmission of the infection. Until vaccination against HSV-2 is available, education to prevent high risk sexual behaviour and the use of condoms appear as preventive measures against HSV-2.
$\mathrm{H}$ erpes simplex virus type 2 (HSV-2) infection has increased in the past few decades in some geographical areas, $^{1-4}$ and has become more relevant given its possible association with risk of acquisition of HIV infection. $^{356}$ Seroprevalence in women is age dependent, and varies from under $20 \%$ to over $40 \%^{7-10}$ in population based studies and up to $90 \%$ in populations with high risk sexual behaviour. ${ }^{8}$ In Latin America, seroprevalences among women has been reported to be $35.8 \%$ in Mexico City $^{10}$ and $42 \%$ in Sao Paulo, Brazil. ' In Costa Rica, a study of women aged 25-59 reported a seroprevalence of $39.4 \% .{ }^{11}$ By contrast, seroprevalence in men is $\sim 25 \%$ lower than in women. ${ }^{28}$

The most consistent risk factors for HSV-2 seropositivity are female sex and cumulative risk of exposure including age, years of sexual activity, and multiple lifetime sexual partners. ${ }^{1}{ }^{211-13}$ Some studies report associations with early sexual debut, ${ }^{9}{ }^{12}{ }^{13}$ parity, ${ }^{13}$ and multiple sexual partners before the first pregnancy. ${ }^{10}$ Others report marital status, lower socioeconomic status, ${ }^{2}{ }^{13}$ limited education, ${ }^{2}$ smoking, vaginal douching, ${ }^{10}$ cocaine use, ${ }^{13}$ and sexually transmitted infections (STI). ${ }^{812}{ }^{13}$ Conversely, condom use has been found to be protective in a few cross sectional ${ }^{911}$ and one prospective study. ${ }^{14}$

True population based surveys describing prevalence and risk factors for HSV-2 antibodies using highly sensitive and specific assays, are rare in developing areas. As part of a worldwide survey of the International Agency for Research on Cancer, we present data from an age stratified sample selected within a population based cohort in Guanacaste (adult female population in 1993 was 66289 ), a rural province of Costa Rica with low HIV incidence rates.

\section{METHODS}

Study population and sample design

One sixth of 1038 censal segments (comprising 40-60 households each) of Guanacaste were randomly selected and, between June 1993 and December 1994, all resident women were invited to participate in a cohort study of the natural history of cervical neoplasia. The study enrolled 10049 women with $93.6 \%$ participation. Details have been described elsewhere. ${ }^{15}$ Briefly, after signing informed consent, participating women were given a questionnaire on sociodemographic, reproductive, contraceptive, sexual, and smoking history. A pelvic examination was performed including collection of exfoliated cervical cells with a Cervex brush (Unimar, CT, USA) for conventional and liquid based cytology (ThinPrep, Cytyc Corporation, MA, USA). Additional cells were collected with a Dacron swab and stored in a DNA transport medium (Virapap, Digene Corporation) for human papillomavirus (HPV) testing. Immediately afterwards the cervix was rinsed twice with $5 \%$ acetic acid and two images of the cervix were taken (Cervigrams, National Testing Laboratories, MI, USA). A $\sim 15 \mathrm{ml}$ heparinised blood sample was drawn, and plasma aliquots were frozen at $-70^{\circ} \mathrm{C}$. Plasma was shipped to the United States on dry ice and stored in liquid nitrogen. Of the 10049 women, 9967 (99.2\%) donated blood and 9175 (96.9\% of sexually active women) underwent a pelvic examination. This analysis is based on an age stratified random sample of 1100 women participating in the enrolment visit. A total of 100 women were randomly selected from each of 11 age strata (18-19 years, 5 year groups to 74 years, and 75 years or older). Plasma samples 
Table 1 Univariate analysis of HSV-2 antibody seroprevalence by selected reproductive and sociodemographic risk factors

\begin{tabular}{|c|c|c|c|c|c|}
\hline Factor & & Number* & $\%$ Positive & OR & $95 \% \mathrm{Cl}$ \\
\hline \multicolumn{6}{|c|}{ Age (years) } \\
\hline & $18-24$ & 141 & 17.7 & 1 & \\
\hline & $25-34$ & 183 & 25.1 & 1.6 & 0.9 to 2.7 \\
\hline & $35-44$ & 195 & 47.7 & 4.2 & 2.5 to 7.1 \\
\hline & $45-54$ & 193 & 53.9 & 5.4 & 3.2 to 9.1 \\
\hline & $55-64$ & 194 & 55.2 & 5.7 & 3.4 to 9.6 \\
\hline & \multirow[t]{2}{*}{$65+$} & 97 & 58.8 & 6.6 & 3.7 to 11.9 \\
\hline & & & & Trend $p<0.001$ & \\
\hline \multicolumn{6}{|c|}{ No of lifetime sexual partners } \\
\hline & 1 & 543 & 28.2 & 1 & \\
\hline & $2-3$ & 356 & 56.5 & 3.3 & 2.5 to 4.4 \\
\hline & \multirow[t]{2}{*}{$4+$} & 104 & 75.0 & 7.6 & 4.7 to 12.4 \\
\hline & & & & Trend $\mathrm{p}<0.001$ & \\
\hline \multicolumn{6}{|c|}{ Education } \\
\hline & None & 116 & 61.2 & 1 & \\
\hline & Primary & 592 & 44.6 & 0.5 & 0.3 to 0.8 \\
\hline & Lower secondary & 109 & 29.4 & 0.3 & 0.2 to 0.5 \\
\hline & Upper secondary & 73 & 34.2 & 0.3 & 0.2 to 0.6 \\
\hline & \multirow[t]{2}{*}{ Post secondary } & 113 & 35.4 & 0.4 & 0.2 to 0.6 \\
\hline & & & & Trend $p<0.001$ & \\
\hline \multicolumn{6}{|c|}{ Marital status } \\
\hline & Married & 732 & 39.8 & 1 & \\
\hline & $\begin{array}{l}\text { Separated/ } \\
\text { divorced }\end{array}$ & 71 & 63.4 & 2.6 & 1.6 to 4.3 \\
\hline & Widowed & 60 & 55.0 & 1.8 & 1.1 to 3.1 \\
\hline & Single & 140 & 45.0 & 1.2 & 0.9 to 1.8 \\
\hline \multicolumn{6}{|c|}{ Smoking } \\
\hline & Never & 875 & 40.5 & 1 & \\
\hline & Former & 78 & 59.0 & 2.1 & 1.3 to 3.4 \\
\hline & Current & 50 & 64.0 & 2.6 & 1.4 to 4.7 \\
\hline & & & & Trend $p<0.001$ & \\
\hline Socioe & status $†$ & & & & \\
\hline & Low & 146 & 49.3 & 1 & \\
\hline & Intermediate & 168 & 37.5 & 0.6 & 0.4 to 1.0 \\
\hline & High & 688 & 43.2 & 0.8 & 0.5 to 1.1 \\
\hline & & & & Trend $p=0.108$ & \\
\hline Age at & ercourse (years) & & & & \\
\hline & 17 & 668 & 38.5 & 1 & \\
\hline & 16 & 335 & 52.2 & 1.7 & 1.3 to 2.3 \\
\hline Numb & births & & & & \\
\hline & $0-2$ & 293 & 35.2 & 1 & \\
\hline & $3-4$ & 253 & 43.9 & 1.4 & 1.0 to 2.0 \\
\hline & $5-7$ & 246 & 50.8 & 1.9 & 1.3 to 2.7 \\
\hline & $8+$ & 152 & 54.6 & 2.2 & 1.5 to 3.3 \\
\hline & & & & Trend $p<0.001$ & \\
\hline Aborti & & & & & \\
\hline & Never & 637 & 40.0 & 1 & \\
\hline & Ever & 307 & 54.4 & 1.8 & 1.4 to 2.4 \\
\hline Cytolo & & & & & \\
\hline & Never & 150 & 37.3 & 1 & \\
\hline & Ever & 852 & 44.0 & 1.3 & 0.9 to 1.9 \\
\hline Hyster & & & & & \\
\hline & Never & 335 & 51.9 & 1 & \\
\hline & Ever & 85 & 62.4 & 1.5 & 0.9 to 2.5 \\
\hline Vagine & & & & & \\
\hline & 4 & 28 & 32.1 & 1 & \\
\hline & 4.5 & 538 & 37.6 & 1.3 & 0.6 to 2.9 \\
\hline & 5 & 261 & 53.3 & 2.4 & 1.0 to 5.5 \\
\hline & 5.5 & 145 & 46.9 & 1.9 & 0.8 to 4.4 \\
\hline & & & & Trend $p<0.001$ & \\
\hline HPV 1 & r 31 serological sta & & & & \\
\hline & Negative & 518 & 35.9 & 1 & \\
\hline & Positive & 485 & 50.7 & 1.8 & 1.4 to 2.4 \\
\hline Oral/i & contraceptive use & & & & \\
\hline & Never & 448 & 46.9 & 1 & \\
\hline & Former & 416 & 42.6 & 0.9 & 0.6 to 1.1 \\
\hline & Current & 139 & 32.4 & 0.5 & 0.4 to 0.8 \\
\hline & & & & Trend $p=0.01$ & \\
\hline HPV D & & & & & \\
\hline & Negative & 755 & 42.0 & 1 & \\
\hline & Positive & 214 & 46.3 & 1.2 & 0.9 to 1.6 \\
\hline Barrier & use & & & & \\
\hline & Never & 619 & 50.1 & 1 & \\
\hline & Former & 285 & 34.0 & 0.5 & 0.4 to 0.7 \\
\hline & Current & 99 & 25.2 & 0.3 & 0.2 to 0.5 \\
\hline & & & & Trend $p<0.001$ & \\
\hline
\end{tabular}

*Women without sexual experience or with missing HSV-2 results are excluded.

$\$ 34$ missing HPV DNA results. 
were selected irrespective of sexual experience and cervical diagnosis.

\section{HSV-2 serological testing}

Plasma samples were shipped to Dr Rhoda Ashley's laboratory for HSV-2 serological testing using type specific HSV-2 and HSV-1 ELISA assays (Focus Technologies, CA, USA). All testing was conducted blinded to other data. All HSV-2 positive sera were retested for confirmation with another Focus Technologies HSV-2 ELISA; 18 specimens without valid result were excluded from analyses.

As a validation of the ELISA, 50 random positive specimens and 50 negative specimens by the HSV-2 ELISA were tested with a western blot test, the current reference standard for HSV-2 serological testing. ${ }^{16}$ The kappa was 0.88 (95\% CI 0.68 to 1.0 ), after exclusion of three pairs with missing results. The ELISA had a sensitivity of $100 \%$, a specificity of $89.5 \%$, a positive predictive value (PPV) of $87 \%$, and a negative predictive value (NPV) of $100 \%$ compared to western blot.

\section{Human papillomavirus (HPV) testing VLP based ELISA}

VLPs were prepared in Trichoplusia ni (High five) cells (Invitrogen, Carlsbad, CA, USA) from recombinant baculoviruses expressing L1 and L2 genes of HPV 16 or 31 or L1 gene alone of HPV 18 and purified by density gradient ultracentrifugation and column chromatography techniques. HPV-16, 18, and 31 specific ELISAs were performed as previously described, ${ }^{17}$ except for the use of an automatic plate washer (Skanwasher 300, Skatron, Norway) and a MultiProbe II robotic liquid handling system (Packard Instruments, CT, USA) to dilute serum samples in $1: 10$ in $0.5 \%$ polyvinyl alcohol (PVA, MW 30 000-70 000, Sigma, St Louis, MO, USA) and $10 \mu \mathrm{l}$ of the diluted serum sample were added to antigen coated plates containing $100 \mu \mathrm{l}$ per well of $0.5 \%$ PVA.

The cut-off point for positive results was determined from the reactivity of concurrently tested plasma samples from self reported virgins. The mean and standard deviation (SD) of optical density (OD) values for controls were calculated and values greater than the mean plus $3 \mathrm{SD}$ were excluded. The analysis was repeated until no further OD values could be excluded by this criterion. A positive cut-off point of $3 \mathrm{SD}$ above the mean of this distribution was chosen. Using this threshold of seropositivity and three HPV types combined (HPV 16, 18, and 31), 97\% of seropositive women were HPV DNA positive for either of the types and among those HPV DNA positive $78 \%$ were seropositive.

\section{Polymerase chain reaction (PCR)}

Details of the MY09/11 Ll consensus primer PCR with AmpliTaq Gold have been previously reported. ${ }^{18-20}$ After an aliquot of the DNA transport medium (Virapap, Digene Corporation) specimen was lysed, the DNA was precipitated by ammonium acetate/ethanol solution, by centrifugation, resuspended in $10 \mathrm{mM}$ TRIS, $\mathrm{pH} 7.5,0.1 \mathrm{mM}$ EDTA, and stored frozen until used. Quality control, amplification, and thermocycling conditions were as previously described..$^{18-20}$

PCR products were analysed by gel electrophoresis and then transferred to Nylon filters. The filters were hybridised overnight with radiolabelled generic probes for HPV (HPV 11 , $16,18,51,73$, and 81 combined) as has been described previously. Dot-blot hybridisation for HPV type specific detection (HPV types 2, 6, 11, 13, 16, 18, 26, 31-35, 39, 40, 42-45, 51-59, 61, 62, 64, 66-70, 71 (AE8), AE2 (IS39), AE9, AE10, and the combination of 2, 13, 34, 42-4, 57, 62, 64, 69, 74,82 (W13B), and AE9) was conducted on specimens that were positive for the generic probes. Three experienced investigators interpreted each dot-blot result, and discrepancies were resolved by consensus.

\section{Statistical analysis}

Overall prevalence in the cohort was estimated using the direct standardisation method with the cohort and the world standard population as reference populations. Univariate and age stratified analyses $(18-24,25-34,35-44,55-64,65+$ years) were carried out to examine associations between HSV-2 seropositivity and the different variables. Odds ratios (ORs) with 95\% exact binomial confidence intervals were calculated and Pearson $\chi^{2}$ test $(p<0.001)$ was used for significance (STATA 7.0, Stata Press, TX, USA). Continuous variables were categorised.

Multivariate logistic regression models, adjusting for lifetime number of sexual partners and age, were then constructed for each risk factor identified in the univariate analysis. To test for statistical significance of dose-response relations $\left(p_{\text {Trend }}\right)$, categorical variables were treated as continuous in the logistic models. The risk factor analysis was restricted to sexually active women with valid HSV-2 results $(n=1003)$. In accordance with the sexual mode of transmission, seroprevalence among virgins was low $(\mathrm{n}=80$, 79 with valid HSV-2 result; $2.5 \%$ ).

\section{RESULTS}

The mean age of the entire cohort was 41.1 years (range 18-97). Among sexually active women, 54\% reported having had only one lifetime sexual partner and $76 \%$ had fewer than three partners. Elementary schooling is common (89\%), but few $(27 \%)$ received 9 or more years of education. Guanacaste is a rural, agricultural province with relatively good public services; $50 \%$ of the women reported to have all household amenities (refrigerator, running water, in-house toilet, electricity, television) and only $9 \%$ declared having none.

The age standardised HSV-2 antibody prevalence for the entire Guanacaste cohort was estimated to be $38.5 \%$ (95\% CI, 37.5 to 39.5 ). The prevalence adjusted for the world standard population was $38.4 \%$ (95\% CI, 38.1 to 38.7 ).

In univariate analysis, age and number of lifetime sexual partners were the major predictors of HSV-2 seropositivity (table 1). Seroprevalence ranged from $17.7 \%$ (95\% CI, 11.8 to 25.0 ) among women $18-24$ years old to $58.8 \%$ (95\% CI, 48.3 to 68.7 ) among those 65 years or older with a 6.6 (95\% CI, 3.7 to 11.9$)$ fold increase in risk $\left(\mathrm{p}_{\text {Trend }}<0.0001\right)$. A strong trend of increasing prevalence was observed with increasing number of sexual partners $\left(\mathrm{p}_{\text {Trend }}<0.0001\right)$. Sexually inexperienced women had a seropositivity of $2.5 \%$ (95\% CI, 0.3 to 8.8 ), while women reporting four or more partners had a seroprevalence of $75 \%$ (95\% CI, 65.6 to 83.0 ) with a 115.5 fold (95\% CI, 26.5-503.4) increase in risk compared to virgins and 7.6 (95\% CI, 4.7 to 12.4 ) compared to monogamous women. Only two HSV-2 seropositive women (0.5\%) reported a history of genital herpetic ulcers.

Additional univariate determinants of HSV-2 seropositivity include limited education, separation or divorce, smoking, low socioeconomic status, sexual initiation before age 17, number of live births, abortion, higher vaginal $\mathrm{pH}$, and seropositivity for HPV 16, 18, or 31. Use of oral/injectable contraceptive methods, having had a cytology, hysterectomy, or being positive for HPV DNA were not significantly associated, but use of barrier methods showed a significant decrease in risk that was even stronger for current users compared to never users (OR 0.3, 95\% CI, 0.2-0.5).

In the multivariate logistic regression, women who were 65 years of age or older were approximately eight times more likely to be HSV-2 seropositive compared to women under 25 years $\left(\mathrm{p}_{\text {trend }}<0.0001\right)$ (table 2 ). A clear trend of increasing risk with increasing number of partners was observed. The age adjusted OR associated with having had four or more sexual partners was 21.4 (95\% CI 7.2 to 63.6) compared to 
Table 2 HSV-2 antibodies adjusted odds ratios (OR) for seropositivity among sexually active women by selected reproductive and sociodemographic risk factors

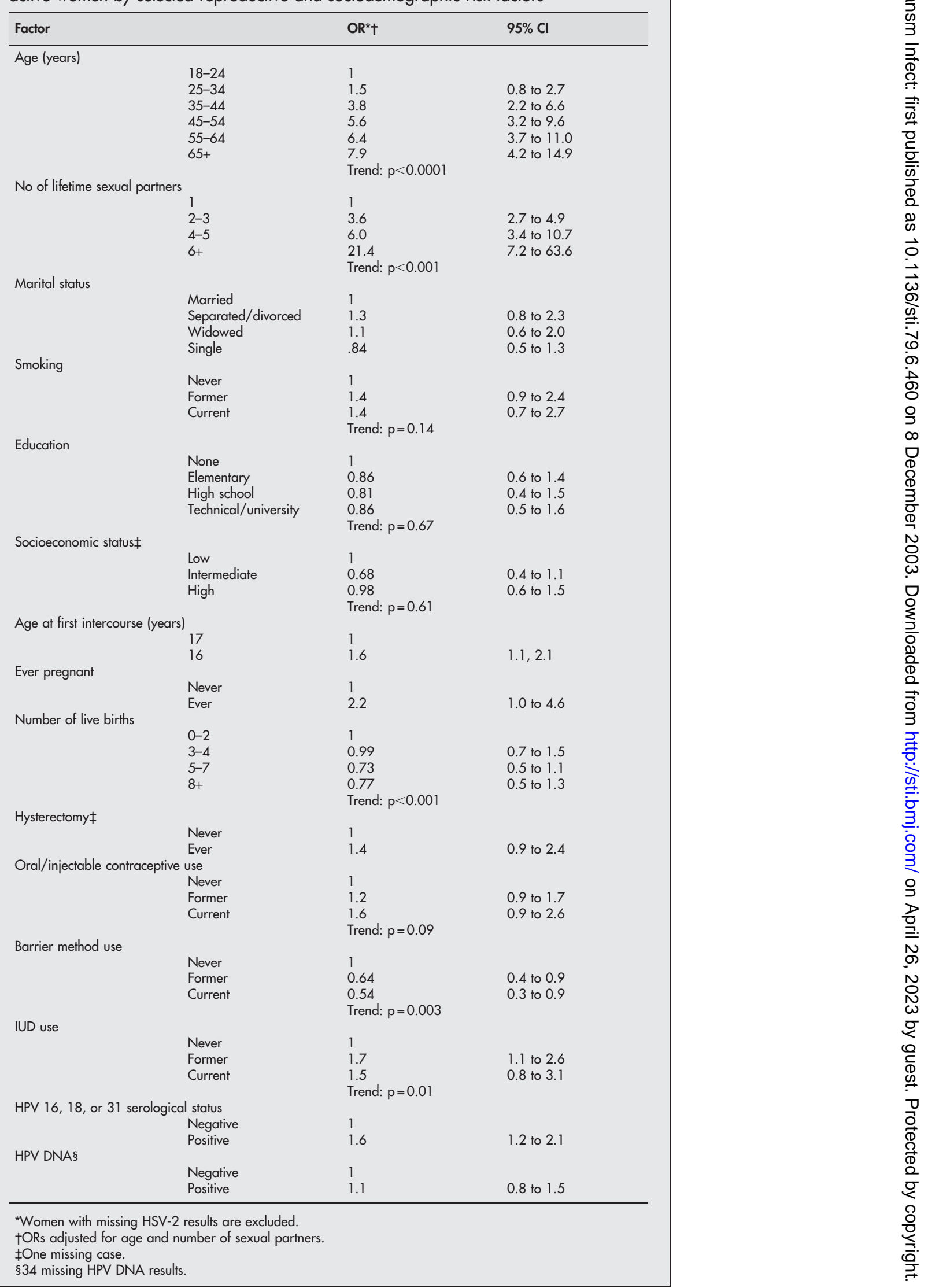


women with one lifetime partner $\left(\mathrm{p}_{\text {Trend }}<0.0001\right)$. Only 10 women reported more than one recent partner and thus this variable could not be evaluated.

The only additional factors that remained statistically significant were age at first intercourse before or at 16 years $(\mathrm{OR}=1.6,95 \% \mathrm{CI}, 1.1$ to 2.1 , compared to those initiating at age 17 or older). Similarly, former intrauterine device users $(\mathrm{OR}=1.7,95 \% \mathrm{CI}, 1.1$ to 2.6 , compared to never users) and HPV 16, 18, or 31 seropositivity (OR $=1.6$; $95 \%$ CI 1.2 to 2.1 ) were significant after adjustment. Barrier methods were protective with a 50\% reduction in detection of HSV-2 antibodies for current (last month) versus never users $(\mathrm{OR}=0.54 ; 95 \% \mathrm{CI}, 0.31$ to 0.94$)$.

Among monogamous women, those separated or divorced had an age adjusted 3.4 (95\% CI 1.4 to 8.2 ) increase in risk of HSV-2 detection.

No statistically significant difference in risk was seen for smoking, education, and socioeconomic status (number of household amenities). Similarly, number of pregnancies, use of oral/injectable contraceptives or positivity for HPV DNA status were not associated with HSV-2 seropositivity.

\section{DISCUSSION}

HSV-2 antibody seroprevalence in Guanacaste (38.5\%) is relatively high for women when compared to other population based studies like the United States (25.6\%; aged $12+$ years) and Spain (3.6\%; age range 5-59). ${ }^{21}$ However, it is consistent with reported seroprevalences from other Latin American countries, $42 \%$ in Brazil $^{9}$ and $35.8 \%$ in Mexico, ${ }^{10}$ although the mean age in this cohort (4l years) was younger than in those two studies. Women from Guanacaste have a low risk self reported sexual behaviour, with only one fourth reporting more than two lifetime sexual partners and only $4.2 \%$ referring having had an STD. The high seroprevalence in the context of low risk female behaviour is probably explained by high risk behaviour of their male partners, as suggested by Oberle et al. ${ }^{11} \mathrm{HSV}-2$ seroprevalence is generally more frequent among women than men, but it is unclear whether women are more susceptible, men are more efficient as vectors, or infected men are less likely to seroconvert. Only one study in Jordan reported higher prevalence in males ${ }^{22}$ and another in Spain reported no difference by sex. ${ }^{21}$ There are no reported HSV-2 studies in Costa Rican men and we had no concurrent information on partners' sexual behaviour.

The previous HSV-2 study in Costa Rican women reported a seroprevalence of $39.4 \%$ in a population with a similar mean age. The provinces of Limón and Puntarenas, which are similar to Guanacaste with regard to rurality and sexual behaviour, ${ }^{23}$ had higher HSV-2 seroprevalence $(\sim 48.7 \%)$ than the rest of the country and also a significant association of HSV-2 seropositivity and being positive for another STD was found. We found no association with a history of STDs or current HPV DNA positivity. The negative association of barrier methods (mainly condoms, other methods are rare in Guanacaste) with HSV-2 seropositivity was consistent in both studies, suggesting that condoms may protect against HSV-2 contagion.

Age and lifetime sexual partners were the major determinants of HSV-2 seropositivity. As expected for any STD, high risk sexual behaviour increases the probability of being exposed. The finding of two HSV-2 seropositive virgins (2.5\%) may reflect non-sexual transmission (that is, perinatal infection, oral) or false positivity of the ELISA used, which was $90 \%$ specific. Among women with multiple sexual partners, HSV-2 prevalence was higher than among monogamous women in all age groups, particularly in the young, and the prevalence peak occurred at earlier ages. In the monogamous group, seroprevalence continued to rise with

\section{Key messages}

- HSV-2 seropositivity is high in Guanacaste, Costa Rica (38.5\%, 95\% Cl 37.5 to 39.5$)$.

- HSV-2 seropositivity in Guanacaste is only a modest sexual behaviour marker for women, given the high seroprevalence in monogamous women $(28.2 \%, 95 \%$ $\mathrm{Cl} 24.4$ to 32.2 ).

- Use of barrier contraceptives was negatively associated with HSV-2 seropositivity (OR $0.54,95 \% \mathrm{Cl}$ 0.31 to 0.94 compared to never users).

- Until vaccination against HSV-2 is available, education to prevent high risk sexual behaviour and the use of condoms appear to be as preventive measures against HSV-2.

age, although less steeply for the older age groups (data not shown). The possibility of HSV-2 being acquired among older age women, an age-cohort effect, or both cannot be ruled out.

In contrast with other studies, education, socioeconomic indicators, number of live births, ever being pregnant, use of oral and injectable hormonal contraceptives were not associated with HSV seropositivity. Although hysterectomy was not associated with an increased risk in the general population, menopausal hysterectomised women had higher HSV-2 seropositivity (data not shown). Although smoking is considered as a possible contributor to viral diseases by altering immune function, ${ }^{24}$ we found no significant association with smoking.

The risk of HSV-2 seropositivity was higher in women with antibodies against a subgroup of high risk HPV types, a partial indicator of HPV exposure. Whether this reflects an interaction that facilitates co-infection or just represents a subgroup at higher risk for both infections is unclear. However, detection of HPV DNA was not associated with HSV-2 seropositivity, probably indicating that HPV DNA represents current infection, which is usually transient and occurs more commonly in younger women.

HSV-2 is an endemic but largely asymptomatic infection in Costa Rican women. Although it is commonly recognised that HSV-2 infection is generally asymptomatic, under-reporting cannot be ruled out. HSV-2 serological screening programmes should be evaluated in selected population groups at especially high risk, either of becoming reservoirs with potential public health impact or in groups at risk of more serious forms of the disease. Identification of antibodies is a reliable method for identifying asymptomatic, never diagnosed population reservoirs for future infections. Pregnant women could be a target group for screening given the silent nature of an infection that can result in neonatal disease.

Control of this viral infection by vaccination may be possible in the future, although many difficulties have to be overcome ${ }^{25}$ in the interim. Use of condoms and education of adolescents, women of reproductive age, and health related personnel on the importance of the diagnosis, treatment, and prevention may help control the spread of HSV-2 infections.

\section{ACKNOWLEDGEMENT}

To the field staff.

\section{CONTRIBUTORS}

ACR was involved in the statistical analysis, interpretation, and manuscript preparation of the HSV-2 study, also in the overall coordination of the cohort follow up; PEC was involved in the initial idea, study design and overall coordination of the HSV-2 study, 
statistical analysis, and manuscript review; JSS was involved in the initial idea, study design, and manuscript review of the HSV-2 study; CB was involved in the design and overall coordination of the cohort and the interpretation and manuscript review of the HSV-2 study; $\mathrm{AH}$ was involved in the design and overall coordination of the cohort, as well as in the initial idea, interpretation, and manuscript review of the HSV-2 study; MS was involved in the design and overall coordination of the cohort, as well as in the initial idea, interpretation, and manuscript review; RV did the HPV serology and manuscript review; RDB did the HPV PCR determination and manuscript review; RA did the HSV-2 serology and manuscript review; XC was involved in the statistical analysis and interpretation and manuscript review; RH was involved in the design and overall coordination of the cohort, as well as in the initial idea, study design, statistical analysis, interpretation and manuscript review of the HSV-2 study.

\section{Authors' affiliations}

A C Rodríguez, C Bratti, R Herrero, Guanacaste Project, Costa Rica Foundation for Health Sciences, San José, Costa Rica P E Castle, A Hildesheim, M Schiffman, Division of Cancer Epidemiology and Genetics, National Cancer Institute, NIH, MD, USA J S Smith, International Agency for Research on Cancer, Lyons, France R Viscidi, Department of Pediatrics, The Johns Hopkins School of Medicine, Baltimore, MD, USA

R D Burk, Cancer Research Center, Albert Einstein College of Medicine, New York, NY, USA

R L Ashley, School of Medicine, University of Washington, Washington DC, USA

X Castellsagué, Instituit Català d'Oncologia, Barcelona, Spain

Financial support: This study was supported by a series of contracts between FUCODOCSA and the National Cancer Institute N01-CP21081, N01-CP-33061, N01-CP-40542, N01-CP-50535, N01-CP81023, and N01-CP-1 1005, National Cancer Institute grant CA78527, Fondo de Investigaciones Sanitarias (FIS), Spain, BAE 01/5013 and a Union International Contre le Cancer Yamagiwa-Yoshida Memorial International Cancer Study Grant.

Informed consent statement: Women who agreed to participate in the population based cohort study of the natural history of cervical neoplasia signed informed consent forms. The protocol was cleared by the institutional review boards of National Cancer Institute, NIH, Maryland, USA and an ad hoc ethics committee of FUCODOCSA, in accordance with the revised Helsinki Declaration of 1983.

\section{REFERENCES}

1 Cusini M, Ghislanzoni M. The importance of diagnosing genital herpes. J Antimicrob Chemother 2001;47(suppl T1):9-16.

2 Fleming DT, McQuillan GM, Johnson RE, et al. Herpes simplex virus type 2 in the United Sates, 1976 to 1994. N Engl J Med 1997;337:1105-11.

3 Nahmias AJ, Lee FK, Beckman-Nahmias S. Sero-epidemiological andsociological patterns of herpes simplex virus infection in the World. Scand J Infect Dis (Suppl) 1990;69:19-36.
4 Corey L, Handsfield $\mathrm{HH}$. Genital herpes and public health: addressing a global problem. JAMA 2000;283:791-4.

5 Schacker T. The role of HSV in the transmission and progression of HIV. Herpes $2001 ; 8: 46-9$.

6 Fleming DT, Wasserheit JN. From epidemiological synergy to public health policy and practice: the contribution of other sexually transmitted diseases to sexual transmission of HIV infection. Sex Transm Infect 1999;75:3-17.

7 Vyse AJ, Gay NJ, Slomka MJ, et al. The burden of infection with HSV-1 and HSV-2 in England and Wales: implications for the changing epidemiology of genital herpes. Sex Transm Infect 2000;76:183-7.

8 Van de Laar MJ, Termorshuizen F, Slomka MJ, et al. Prevalence and correlates of herpes simplex virus type 2 infection: evaluation of behavioural risk factors. Int J Epidemiol 1998;27:127-34.

9 Smith J, Herrero R, Munoz N, et al. Prevalence and risk factors for herpes simplex virus type 2 infection among middle-aged women in Brazil and The Philippines. Sex Transm Dis 2001;28:187-94.

10 Lazcano-Ponce E, Smith JS, Muñoz N, et al. High prevalence of antibodies to herpes simplex virus type 2 among middle-aged women in Mexico City, Mexico: a population-based study. Sex Transm Dis 2001;28:270-6.

11 Oberle M, Rosero-Bixby L, Lee F, et al. Herpes simplex virus type 2 antibodies: high prevalence in monogamous women in Costa Rica. Am J Trop Med Hyg 1989;41:224-9.

12 Austin H, Macaluso M, Nahmias A, et al. Correlates of herpes simplex virus seroprevalence among women attending a sexually transmitted disease clinic. Sex Transm Dis 1999;26:329-34.

13 Buchacz K, McFarland W, Hernández M, et al. Prevalence and correlates of herpes simplex virus type 2 infection in a population-based survey of young women in low-income neighborhoods of Northern California. The Young Women's Survey Team. Sex Transm Dis 2000;27:393-400.

14 Wald A, Langenberg AG, Link K, et al. Effect of condoms on reducing the transmission of herpes simplex virus type 2 from men to women. JAMA 2001;285:3100-6.

15 Herrero R, Schiffman MH, Bratti C, et al. Design and methods of a populationbased natural history study of cervical neoplasia in a rural province of Costa Rica: the Guanacaste Project. Pan Am J Public Health 1997; 1:362-75.

16 Ashley RL. Sorting out the new HSV type specific antibody tests. Sex Transm Infect 2001;77:232-7.

17 Studentsov YY, Schiffman M, Strickler HD, et al. Enhanced enzyme-linked immunosorbent analysis for detection of antibodies to virus-like particles of human papillomavirus. J Clin Microbiol 2002;40:1755-60.

18 Castle PE, Schiffman M, Gravitt PE, et al. Comparison of HPV DNA detection by MY09/11 PCR methods. J Med Virol 2002;68:417-23.

19 Qu W, Jiang G, Cruz Y, et al. PCR detection of human papillomavirus: comparison between MYO9/MY11 and GP5+/GP6+primer systems. J Clin Microbiol 1997; 35:1304-10.

20 Burk RD, Ho GY, Beardsley L, et al. Sexual behaviour and partner characteristics are the predominant risk factors for genital human papillomavirus infection in young women. J Infect Dis 1996;174:679-89.

21 Garcia-Corbeira P, Dal-Re R, Aguilar L, et al. Is sexual transmission an important pattern for herpes simplex type 2 virus seroconversion in the Spanish general population? J Med Virol 1999:59:194-7.

22 Abuharfeil N, Meqdam MM. Seroepidemiologic study of herpes simplex virus type 2 and cytomegalovirus among young adults in northern Jordan. New Microbiol 2000;23:235-9.

23 Herrero $R$, Brinton $L$, Hartge $P$, et al. Determinants of the geographic variation of invasive cervical cancer in Costa Rica. Pan Am J Public Health 1993;27:15-25.

24 Sopori ML, Kozak W. Immunomodulatory effects of cigarette smoke. J Neuroimmunol 1998;83:148-56.

25 Stanberry LR, Spruance SL, Cunningham AL, et al. Glycoprotein-D-adjuvant vaccine to prevent genital herpes. N Engl J Med 2002;347:1652-61. 